\title{
Prevalence and Clinical Significance of Mycoplasma genitalium in Gynecologic Patients
}

\author{
Oluwatosin Goje ${ }^{1 *}$, Jessian L Munoz ${ }^{1}$, Frederick S Nolte ${ }^{2}$ and David E Soper ${ }^{3}$ \\ ${ }^{1}$ Obstetrics, Gynecology and Women's Health Institute, Cleveland Clinic Desk, Cleveland, USA \\ ${ }^{2}$ Pathology and Laboratory Medicine, Medical University of South Carolina, Charleston SC, USA \\ ${ }^{3}$ Department of Obstetrics and Gynecology, Medical University of South Carolina, Charleston SC, USA
}

\begin{abstract}
Objective: Mycoplasma genitalium has been recognized as a cause of male urethritis, and there is now evidence suggesting it causes cervicitis and pelvic inflammatory disease (PID) in women.

Methods: Prevalence, risk factors and co-infections with other sexually transmitted pathogens were collected in a cross-sectional study looking at 400 women at the gynecologic clinics of a university medical center in the United States. Bacterial vaginosis and trichomoniasis were diagnosed using Amsel's criteria, gram stain and trichomonas culture respectively. Cervicitis and PID were clinically diagnosed. After testing for Chlamydia trachomatis and Neisseria gonorrhoeae, the residual cervical swab transport medium (Gen-Probe/Hologic ${ }^{\circledR}$ ) was stored at $-70^{\circ} \mathrm{C}$. Stored samples were later analyzed for $M$. genitalium by a research use only transcription-mediated amplification assay using procedures similar to those established for APTIMA Combo2 assay for C. trachomatis and N. gonorrhoeae (Gen-Probe/Hologic ${ }^{\circledR}$ ).
\end{abstract}

Results: The overall prevalence of infection with C. trachomatis, N. gonorrhoeae, T. vaginalis and M. genitalium was found to be $7.8 \%, 1.8 \%, 10.43 \%$ and $8.9 \%$, respectively. Prevalence of $M$. genitalium was comparable to that of $C$. trachomatis and greater than the prevalence of $N$. gonorrhoeae. Univariate analysis of $M$. genitalium status showed that participants with lower condom use had an increased probability of M. genitalium ( $p=0.037)$.

Conclusion: Prevalence of $M$. genitalium was comparable to $C$. trachomatis in our study, but more research is needed to clarify pathogenicity.

Keywords: M. genitalium; C. trachomatis; N. gonorrhoeae; Risk factors

\section{Introduction}

Although Mycoplasma genitalium is well documented as a causative pathogen in non-gonococcal, non-chlamydial urethritis in men; the manifestations of infection in women are less well described. There is now evidence suggesting it causes cervicitis and pelvic inflammatory disease (PID) in women [1]. One of the major limitations encountered in the study of $M$. genitalium is that it is fastidious and difficult to culture. Although there is no U.S food and drug administration (FDA) approved commercial detection system, the availability of molecular methods for research and commercial purposes has altered our ability to derive valid information about the pathogenicity of this bacterium. There have been more studies in recent years researching into its pathogenicity and treatment [2-6]. The most recent Centers for Disease Control and Prevention (CDC) Sexually Transmitted Diseases treatment guidelines discussed it under emerging issues [1]. M. genitalium has been implicated in endometritis, cervicitis and pelvic Inflammatory Diseases (PID) and it may have significant effect on reproductive health and pregnancy outcomes $[7,8]$.

Therefore, the purpose of this study was to investigate the prevalence of M. genitalium in women aged 18 and older attending our clinic, determine the percentage of women diagnosed with cervicitis and PID who were M. genitalium positive. We wanted to identify women at high risk for $M$. genitalium and examine the relationship between $M$.genitalium and other known sexually transmitted infections (STIs).

\section{Methods}

A cross-sectional study was performed following the approval of the study protocol by the Institutional Review Board (IRB) at a tertiary university medical center. Participants who presented to the Obstetrics and Gynecology clinics for care were included if they were 18 years or older and sexually active. Participants who met criteria were consented, enrolled and interviewed by the research coordinator. History, pelvic and speculum examination, cervical and vaginal samples were collected by the health care provider performing the gynecologic exam.

Saline microscopy to diagnose bacterial vaginosis (BV), candidiasis and Trichomonas vaginalis was performed and documented by the health care provider. Diagnosis of PID was made based on the CDC guidelines [1]. A patient was diagnosed with cervicitis if the clinician found: mucopurulent cervical discharge/mucopus and/or friable cervix that bled easily on application of a swab. The principal investigator confirmed the diagnosis of PID by reviewing the data collected by physicians. For this study, a T. vaginalis culture was performed using the In-Pouch system, BV diagnosis was initially made using Amsel's criteria and confirmed with gram stain. Gonorrhea and or Chlamydia infection was diagnosed using nucleic acid amplification test (NAAT). An endocervical swab contained in the APTIMA Unisex swab specimen Collection Kit was used to collect patient swab specimen per manufacturer's protocol and was processed for gonorrhea and chlamydia NAAT.

*Corresponding author: Oluwatosin Goje, Obstetrics, Gynecology and Women's Health Institute, Cleveland Clinic, Desk A81, 9500 Euclid Avenue, Cleveland, $\mathrm{OH}$ 44195, USA; Tel: 440-315-3809; E-mail: Gojeo@ccf.org

Received May 11, 2017; Accepted May 17, 2017; Published May 24, 2017

Citation: Goje O, Munoz JL, Nolte FS, Soper DE (2017) Prevalence and Clinical Significance of Mycoplasma genitalium in Gynecologic Patients. J AIDS Clin Res 8 : 694. doi: 10.4172/2155-6113.1000694

Copyright: ( 2017 Goje O, et al. This is an open-access article distributed under the terms of the Creative Commons Attribution License, which permits unrestricted use, distribution, and reproduction in any medium, provided the original author and source are credited. 


\section{M. genitalium transcription-mediated amplification (TMA) assay}

After testing for Chlamydia trachomatis and Neisseria gonorrhoeae, the residual cervical swab transport medium (Gen-Probe/Hologic") was stored at $-70^{\circ} \mathrm{C}$. The specimens were analyzed for $M$. genitalium by target capture, amplification by TMA and detection by the hybridization protection assay in a manner similar to procedures established for APTIMA Combo2 assay kit protocols established for C. trachomatis and N. gonorrhoeae (GenProbe/Hologic ${ }^{\circ}$ ). Primers and probes and target capture oligonucleotides were designed by Gen-Probe/Hologic to be specific for M. genitalium and were designed to be used with the reagents that the same formulation as APTIMA Combo2 as previously described $[9,10]$.

The M. genitalium assay was performed on the Tigris system. The reagents were provided to us for research use only. The threshold for positive reactions was set at $\geq 40,000$ relative light units [10]. This cutoff was validated in our laboratory with a panel of 25 positive and 25 negative specimens previously tested at the University of North Carolina at Chapel Hill with the same M. genitalium TMA assay. The positive and negative percent agreement between the results of the tests was $92 \%$ and $100 \%$, respectively.

\section{Statistical methods}

The documented prevalence of $M$. genitalium in literature varies; it depends on communities and clinics where research was performed. We assumed a $20 \%$ prevalence of $M$. genitalium similar to Gaydos et al.s Baltimore study [11]. Numerical measures was summarized by mean and standard deviation when the values are approximated by a normal distribution; otherwise summarized by median and interquartile range. Normality determined by visual inspection of histograms and normal QQ plots. Categorical values summarized by frequency and percentage. Statistical methods to each study aim are described below.

Prevalence of M. genitalium estimated as the proportion observed in the sample with a $95 \%$ confidence interval based on a normal approximation of the proportion. Potential risk factors for M. genitalium determined from univariable comparisons of potential risk factors with $M$. genitalium. Comparisons performed by t-test, Wilcoxon test, or chi-square test of association, depending on the level of measurement of the potential risk factor. Associations of morbidity (cervicitis and PID) evaluated using univariable chi-square tests of association. Associations of M. genitalium with gonorrhea, chlamydia, and bacterial vaginosis and trichomonas infection evaluated using univariable chi-square tests of association.

\section{Results}

A total of 400 women participated in the study with majority identifying as African American (71.8\%) and unmarried (93.5\%). Majority of the cohort were non-smokers (77.5\%). $76.5 \%$ selfreported sexual debut by age 18 years, $93.6 \%$ had male sex partners. Majority (80.25\%) of the cohort agreed that condom use during sexual intercourse prevents STI but only $19.75 \%$ consistently used condoms during sexual intercourse. In our cohort, $9 \%$ never used condoms during sexual intercourse. There was a past history of STI in our cohort, with $40.5 \%$ endorsing a past history of chlamydia, and $5.75 \%$ being HIV infected. Vaginal discharge followed by abdomino-pelvic pain were the most commonly reported symptoms ( $28.25 \%$ and $14.5 \%$, respectively) and rate of cervicitis and PID were $11 \%$ and $7 \%$, respectively.

The overall prevalence of infection with C. trachomatis, $N$. gonorrhoeae, T. vaginalis and M. genitalium was found to be $7.8 \%, 1.8 \%$, $10.43 \%$ and $8.9 \%$, respectively.

There was no difference in the median age between $M$. genitalium positive and negative participants; 25 years versus 24 years. $(\mathrm{p}=0.84)$. Majority of M. genitalium positive patients self-identified as African Americans and were unmarried (83.3\% and $91.7 \%$, respectively). There was no difference in race, marital status, educational level or tobacco history between $M$. genitalium positive women and $M$. genitalium negative women. There was no difference in sex partner preference and total lifetime partners between both groups.

Twenty four of the participants screened positive for M. genitalium, the percentage was $8.9 \%$ with a $95 \%$ confidence interval of $(5.9 \%$, $13.1 \%)$. Looking at the M. genitalium cohort, $79.2 \%$ did not smoke cigarette, $62.5 \%$ had sexual debut between $15-18$ years of age and all had male sex partners. $45.8 \%$ had a previous history of chlamydia, $16.7 \%$ history of gonorrhea and $12.5 \%$ were HIV positive. Based on presenting symptoms, $20.8 \%$ complained of vaginal discharge and $20.8 \%$ pelvic or lower abdominal pain. $8.3 \%$ complained of dyspareunia, $4.2 \%$ complained of vaginal spotting/bleeding (Table 1 ). None of the

\begin{tabular}{|c|c|c|c|c|c|c|}
\hline Symptoms & Total & $\mathrm{N}$ (M. gen negative) & Percent & N (M. gen positive) & Percent & P-value (f) \\
\hline Vaginal Discharge & 400 & & & & & 0.63 \\
\hline No & 287 & 176 & 72.13 & 19 & 79.17 & \\
\hline Yes & 113 & 68 & 27.87 & 5 & 20.83 & \\
\hline Burning on urination & 400 & & & & & $>0.99$ \\
\hline No & 398 & 243 & 99.59 & 24 & 100 & \\
\hline Yes & 2 & 1 & 0.41 & 0 & 0 & \\
\hline Lower abdominal/pelvic pain & 400 & & & & & 0.35 \\
\hline No & 342 & 212 & 86.89 & 19 & 79.17 & \\
\hline Yes & 58 & 32 & 13.11 & 5 & 20.83 & \\
\hline Pain with sexual intercourse & 400 & & & & & $>0.99$ \\
\hline No & 361 & 218 & 89.34 & 22 & 91.67 & \\
\hline Yes & 39 & 26 & 10.66 & 2 & 8.33 & \\
\hline Vulvo-vaginal itching & 400 & & & & & $>0.99$ \\
\hline No & 398 & 243 & 99.59 & 24 & 100 & \\
\hline Yes & 2 & 1 & 0.41 & 0 & 0 & \\
\hline Vaginal Bleeding & 400 & & & & & 0.70 \\
\hline No & 367 & 223 & 91.39 & 23 & 95.83 & \\
\hline Yes & 33 & 21 & 8.61 & 1 & 4.17 & \\
\hline
\end{tabular}

f: Fisher's exact test for count data

Table 1: M. genitalium was not associated with any specific gynecologic symptoms. 


\begin{tabular}{|c|c|c|c|c|c|c|}
\hline Factor & Total & $\mathrm{N}$ (M. gen negative) & Percent & N (M. gen positive) & Percent & $p$-value (f) \\
\hline Race & 400 & & & & & 0.11 \\
\hline African American & 287 & 161 & 65.98 & 20 & 83.33 & \\
\hline Other & 113 & 83 & 34.02 & 4 & 16.67 & \\
\hline Use of Condoms & 392 & & & & & 0.81 \\
\hline No & 99 & 58 & 24.58 & 5 & 20.83 & \\
\hline Yes & 293 & 178 & 75.42 & 19 & 79.17 & \\
\hline History of condom use & 400 & & & & & 0.037 \\
\hline Never & 35 & 15 & 6.53 & 5 & 20.83 & \\
\hline Sometimes & 137 & 89 & 36.33 & 6 & 25 & \\
\hline Most of the time & 149 & 96 & 39.18 & 6 & 25 & \\
\hline Always & 79 & 44 & 17.96 & 7 & 29.17 & \\
\hline
\end{tabular}

f: Fisher's exact test for count data

Table 2: Univariable comparisons by Mycoplasma status.

participants complained of dysuria, increased urinary frequency or vaginal itching. We also looked at the association of M. genitalium with other STIs diagnosed during the same gynecologic visit (co-infection), and $4.2 \%$ of M. genitalium patients had gonorrhea, $8.3 \%$ had chlamydia, $12.6 \%$ trichomonas and $58.3 \%$ had BV in addition to M. genitalium. One M. genitalium patient was diagnosed with PID and 4 diagnosed with cervicitis.

Only one risk factor showed a significant relationship with $M$. genitalium in a univariate comparison by mycoplasma status (Table 2). Participants with lower condom use had an increased probability of $M$. genitalium ( $\mathrm{p}=0.037)$. There was no association between the presence of $M$. genitalium and a previous history of STIs. When we looked at the relationship of M. genitalium and other STIs in our study, it appears that $M$. genitalium develops independently of these infections. The most common co-infection was BV (58.3\%).

\section{Discussion}

The median age of M. genitalium participants was $25(22,27)$ years and majority of the participants were African American (83.3\%) and single (91.7\%). Our result agrees with the demographic pattern of M. genitalium and other STIs as described in other studies $[11,12]$. Patients with STIs tend to be younger and African Americans are disproportionately affected. Oakeshott et al. [12] demonstrated young age and race as risk factors for M. genitalium, in addition, majority of patients in the Baltimore study were African American. Olsen et al. had a very low prevalence of $M$. genitalium among married Vietnamese women [13].

The prevalence of $M$. genitalium (8.9\%) was comparable to that of C. trachomatis (7.8\%), which was one of our hypotheses; and greater than the prevalence of gonorrhea, which was $1.8 \%(\mathrm{p}=0.017)$. The prevalence of $M$. genitalium has been reported to range from $0 \%$ to $40 \%$ in the literature depending on the clinic/site that was sampled and the type of specimen collected (i.e., vaginal vs. cervical vs. urine samples) [14,15]. Gaydos et al. [11] reported M. genitalium prevalence of $19.2 \%$ in a Baltimore STD clinic; in the PEACH study, approximately $15 \%$ of women were infected with M. genitalium, $14 \%$ were infected with C. trachomatis and 15\% were infected with N. gonorrhoeae [16]. Conversely, Oakeshott et al. [12] reported a baseline prevalence of 3.3\% in the community. A national longitudinal study of adolescent health showed that genital prevalence of $M$. genitalium (approximately 1\%) was approximately between those of $N$. gonorrhoeae $(0.4 \%)$ and $C$. trachomatis (4.2\%) [14]. When we looked at history of previous STIs, there was no significant relationship between $M$. genitalium and other STIs.
A significant observation was that subjects with lower condom use had increased probability of M. genitalium ( $\mathrm{p}=0.037$ ). Condom use, which would be expected to be protective against $M$. genitalium infection if sexual transmission were the main route of transmission was measured in only four studies and these, provided conflicting information. Two of these studies found no association between condom use and $M$. genitalium infection, the remaining two studies reported opposite results $[17,18]$.

Among West African commercial sex workers, women who reported condom use with all clients had less $M$. genitalium infection when compared with women who did not use condoms all the time ( $24.3 \%$ vs. $33.0 \%, P=0.02$ ) [19]. In contrast, among young adults in the National Longitudinal Study of Adolescent Health, condom use was associated with an increased prevalence of M. genitalium [14]. Although these disparate results suggest condoms may not be effective against M. genitalium infection, condom use data may be difficult to interpret.

There was no significant difference in presenting symptoms and no difference in the rates of PID and cervicitis among the different STIs. Our results differ from that of other studies which found an increase in cervicitis and PID among patients with $M$. genitalium infection [11,12,20]. Bjartling et al. [20] demonstrated M. genitalium was an independent and strong risk factor for both cervicitis and PID although compared to C. trachomatis, clinical manifestations were less frequent. On the other hand, our study agrees with that of Tosh et al. [21], the authors found that women identified with M. genitalium in a primary care center were no more symptomatic than uninfected women [21]. Manhart et al. [14] also found that lower genital tract; M. genitalium infection was not associated with symptoms.

Overall, $28.3 \%$ of our patients presented with abnormal vaginal discharge but there was no difference between $M$. genitalium positive and negative participants when vaginal discharge was analyzed. Results are mixed about the role of vaginal discharge in M. genitalium infection; some studies found no correlation between $M$ genitalium and vaginal discharge while others did demonstrate a relationship. Vaginal discharge was more common in women with lower genital tract M. genitalium infections compared to women without M. genitalium among 390 minority women attending a public health clinic [22]. Vandepitte et al. [23] found that urethritis and mucopurulent vaginal discharge were associated with M. genitalium infection in a group at high risk for HIV and other STIs in Uganda.

Bacterial vaginosis was the most common detectable co-infection with M. genitalium in our study. This agrees with the UK community based study where presence of BV was a risk factor for M. genitalium. 
Citation: Goje O, Munoz JL, Nolte FS, Soper DE (2017) Prevalence and Clinical Significance of Mycoplasma genitalium in Gynecologic Patients. J AIDS Clin Res 8: 694. doi: 10.4172/2155-6113.1000694

Page 4 of 4

Results of co-infection in M. genitalium studies are also mixed [11,16,2426]. While the PEACH study and Baltimore study documented a high rate of co-infection $[11,16]$, other studies reported little or none $[24,26]$.

\section{Conclusion}

Mycoplasma genitalium was as prevalent as chlamydia in our study. The clinical spectrum of M. genitalium infection has been reported to be similar to that observed with chlamydial infection. Although not significantly associated with morbidity (cervicitis and PID) in our study, our study was limited by the number of patients who tested positive for M. genitalium.

Sexually transmitted infections are prevalent public health issues. In women, STI can cause PID, a significant public issue in the United States and worldwide. Adverse sequelae, including tubal factor infertility, chronic pelvic pain, recurrent PID and ectopic pregnancy can occur if PID treatment is delayed or avoided. Although PID an ascending infection is polymicrobial, M. genitalium has been identified as a possible etiologic agent, and our study has shown that M. genitalium is as prevalent as chlamydia. Due to the asymptomatic nature, like chlamydia, $M$. genitalium may go undetected, left untreated, and subsequently progress to reproductive morbidity.

\section{Limitations}

Our study is single-centered with small sample size of M. genitalium patients. We were unable to detect major differences in our univariate analyses.

\section{Acknowledgement}

- Gen-Probe/Hologic for supplying the reagents and assay for Mycoplasma genitalium TMA.

- The research laboratory of Dr. Marcia Hobbs, University of North Carolina, Chapel Hill, NC for technical support.

- The Molecular Pathology Laboratory of the Medical University of South Carolina, Charleston, SC for validating and performing the Mycoplasma. genitalium TMA research assay.

\section{References}

1. Workowski KA, Bolan GA; Centers for Disease C and Prevention (2015) Sexually transmitted diseases treatment guidelines. MMWR Recomm Rep 64: 1-137.

2. Le Roy C, Pereyre S, Bebear C (2014) Evaluation of two commercial real-time PCR assays for detection of Mycoplasma genitalium in urogenital specimens. $\mathrm{J}$ Clin Microbiol 52: 971-973.

3. Takanashi M, Ito S, Kaneto H, Tanahashi Y, Kitanohara M, et al. (2015) Development and clinical application of an InvaderPlus $(R)$ assay for the detection of genital mycoplasmas. J Infect Chemother 21: 516-519.

4. Twin J, Jensen JS, Bradshaw CS, Garland SM, Fairley CK, et al. (2012) Transmission and selection of macrolide resistant Mycoplasma genitalium infections detected by rapid high resolution melt analysis. PLoS ONE 7: e35593.

5. Peuchant O, Menard A, Renaudin H, Morozumi M, Ubutkata K, et al. (2009) Increased macrolide resistance of Mycoplasma pneumoniae in France directly detected in clinical specimens by real-time PCR and melting curve analysis. J Antimicrob Chemother 64: 52-58.

6. Touati A, Peuchant O, Jensen JS, Bebear C, Pereyre S (2014) Direct detection of macrolide resistance in Mycoplasma genitalium isolates from clinical specimens from France by use of real-time PCR and melting curve analysis. $J$ Clin Microbiol 52: 1549-1555.

7. Manhart LE, Critchlow CW, Holmes KK, Dutro SM, Eschenbach DA, et al.
(2003) Mucopurulent cervicitis and Mycoplasma genitalium. J Infect Dis 187 650-657.

8. Munoz JL, Goje OJ (2016) Mycoplasma genitalium: An emerging sexually transmitted infection. Scientifica 2016: 7537318.

9. Hardick J, Giles J, Hardick A, Hsieh YH, Quinn T, et al. (2006) Performance of the gen-probe transcription-mediated amplification research assay compared to that of a multitarget real-time PCR for Mycoplasma genitalium detection. J Clin Microbiol 44: 1236-1240.

10. Wroblewski JK, Manhart LE, Dickey KA, Hudspeth MK, Totten PA (2006) Comparison of transcription-mediated amplification and PCR assay results for various genital specimen types for detection of Mycoplasma genitalium. J Clin Microbiol 44: 3306-3312.

11. Gaydos C, Maldeis NE, Hardick A, Hardick J, Quinn TC (2009) Mycoplasma genitalium as a contributor to the multiple etiologies of cervicitis in women attending sexually transmitted disease clinics. Sex Transm Dis 36: 598-606.

12. Oakeshott P, Aghaizu A, Hay P, Reid F, Kerry S, et al. (2010) Is Mycoplasma genitalium in women the "New Chlamydia?" A community-based prospective cohort study. Clin Infect Dis 51: 1160-1166.

13. Olsen B, Lan PT, Stalsby Lundborg C, Khang TH, Unemo M (2009) Populationbased assessment of Mycoplasma genitalium in Vietnam--low prevalence among married women of reproductive age in a rural area. J Eur Acad Dermatol Venereol 23: 533-537.

14. Manhart LE, Holmes KK, Hughes JP, Houston LS, Totten PA (2007) Mycoplasma genitalium among young adults in the United States: An emerging sexually transmitted infection. Am J Public Health 97: 1118-1125.

15. McGowin CL, Anderson-Smits C (2011) Mycoplasma genitalium: An emerging cause of sexually transmitted disease in women. PLoS Pathog 7:e1001324.

16. Haggerty CL, Totten PA, Astete SG, Lee S, Hoferka SL, et al. (2008) Failure of cefoxitin and doxycycline to eradicate endometrial Mycoplasma genitalium and the consequence for clinical cure of pelvic inflammatory disease. Sex Transm Infect 84: 338-342.

17. Huppert JS, Mortensen JE, Reed JL, Kahn JA, Rich KD, et al. (2008) Mycoplasma genitalium detected by transcription-mediated amplification is associated with Chlamydia trachomatis in adolescent women. Sex Transm Dis 35: 250-254.

18. Hamasuna R, Imai H, Tsukino H, Jensen JS, Osada $Y$ (2008) Prevalence of Mycoplasma genitalium among female students in vocational schools in Japan. Sex Transm Infect 84: 303-305

19. Pepin J, Labbe AC, Khonde N (2005) Mycoplasma genitalium: An organism commonly associated with cervicitis among west African sex workers. Sex Transm Infect 81: 67-72.

20. Bjartling C, Osser S, Persson K (2012) Mycoplasma genitalium in cervicitis and pelvic inflammatory disease among women at a gynecologic outpatient service. Am J Obstet Gynecol 206: e1-e8.

21. Tosh AK, Van Der Pol B, Fortenberry JD, Williams JA, Katz BP, et al. (2007) Mycoplasma genitalium among adolescent women and their partners. J Adolesc Health 40: 412-417.

22. Korte JE, Baseman JB, Cagle MP, et al. (2006) Cervicitis and genitourinary symptoms in women culture positive for Mycoplasma genitalium. Am J Reprod Immunol 55: 265-275.

23. Vandepitte J, Bukenya J, Hughes P, Muller E, Buvé A, et al. (2012) Clinical characteristics associated with Mycoplasma genitalium infection among women at high risk of HIV and other STI in Uganda. Sex Transm Dis 39: 487-491.

24. Simms I, Eastick K, Mallinson H, Thomas K, Gokhale R, et al. (2003) Associations between Mycoplasma genitalium, Chlamydia trachomatis and pelvic inflammatory disease. J Clin Pathol 56: 616-618.

25. Cohen CR, Manhart LE, Bukusi EA, Astete S, Brunham RC, et al. (2002) Association between Mycoplasma genitalium and acute endometritis. Lancet 359: 765-766.

26. Cohen CR, Mugo NR, Astete SG, Odondo R, Manhart L, et al. (2005) Detection of Mycoplasma genitalium in women with laparoscopically diagnosed acute salpingitis. Sex Transm Infect 81: 463-466. 